\title{
Study on the Value of Yunnan Maca in Sports Nutrition
}

\author{
Tong Jinfeng \\ Career Academy College of Wuhan City occupation teacher
}

\begin{abstract}
Through the scientific analysis of the nutrition data of the plant Maca the paper do the research into value of Maca in the sport nutrition, The research indicafes the sport player take the Maca in the sport diet, which will .help athlete's recovery and be good to get better place in the gane.
\end{abstract}

Key words - sport nutrition, value of Maca

\section{云南玛卡在运动营养学中的价值研究}

\author{
童锦锋 \\ 武汉城市职业学院职业网球学院教师
}

\begin{abstract}
摘 要 通过对已知玛卡营养数据科学分析, 结合运动营养学中知识, 来体现玛卡在运动营养学中的研究价值。研究表明: 如果 在运动员的饮食调理搭配中, 科学运用玛卡这种药材, 对于运动员身体的恢复和成绩的提高能起到非常好的效果, 在实际运动营养学 研究中具有很好研究价值。
\end{abstract}

关键词 玛卡, 运动营养学, 价值研究

玛卡, 学名 maca。玛卡是十字花科的高原植物, 原产 于南美秘鲁安第斯山 4000 米以上的高原植物。玛卡独含有 的玛卡酰胺和玛卡稀对平衡人体荷尔蒙分泌有显著的效 果。玛卡作为具有很强的营养成分, 具有提高人体免疫力、 快速恢复人体体力、提高抗疲劳能力, 医学研究正常使用 对人体无副作用。由于其很好的医用价值及经济价值, 以 及海拔气候等自然条件的相似性, 我国西部云贵高原一些 地方引进玛卡进行试验种植, 并获得成功。科学人员还对 我国云南种植玛卡进行科学的营养成分的分析研究, 为深 入了解玛卡提供重要的科学数据。

\section{1. 研究对象与方法}

\section{1 研究对象}

对玛卡的营养成分在运动营养学中价值进行研究。

\section{2 研究方法}

1.2.1 本文通过文献资料发, 查阅玛卡的大量营养成分资料。

\section{2 .2 采用数理统计法}

通过其他学者对玛卡营养成分科学数据, 结合运动营 养学相关知识进行分析。

\section{2. 玛卡营养成分测定}

表 1 云南玛卡的主要营养成分

\begin{tabular}{|c|c|c|c|c|c|c|c|}
\hline 项目 & $\begin{array}{c}\text { 蛋白 } \\
\text { 质 } / \%\end{array}$ & $\begin{array}{c}\text { 脂肪 } \\
1 \%\end{array}$ & $\begin{array}{c}\text { 粗纤 } \\
\text { 维 } / \%\end{array}$ & $\begin{array}{c}\text { 碳水化 } \\
\text { 物 } / \%\end{array}$ & $\begin{array}{c}\text { 热值 } \\
1 \%\end{array}$ & $\begin{array}{c}\mathrm{VC} \\
/(\mathrm{mg} / \mathrm{kg})\end{array}$ & $\begin{array}{c}\text { 灰分 } \\
1 \%\end{array}$ \\
\hline 测定值 & 12.31 & 0.92 & 32.57 & 51.2 & 16464 & 314.97 & 3.08 \\
\hline
\end{tabular}

2.1.1 通过实验对玛卡的营养学分析数据可知每 1 克玛卡中 蛋白质含量占 $12.31 \%$, 而蛋白质与人体体内生理功能有 关, 能维持体内酸碱平衡的作用。玛卡的蛋白质的含量是 非常高的, 在营养学中认为食物体中蛋白质的高与低是衡 量食物营养价值的重要数据指标。

2.1.2 在表中可以看到碳水化合物在物体中所占比例为 $51.2 \%$, 占到总质量的一半以上。水可以维持运动员在身体 体内电解质平衡, 并且起到良好的物质溶解, 加快生化反 应, 调节运动员体温作用, 并能对运动员关节起到润滑作 用。

2.1.3 玛卡的热值含量很高, 达到 $16464 \mathrm{~J} / \mathrm{g}$. 而热量为运动员 提供能量和保持充沛的运动能力。 
2.1.4 玛卡的 VC 含量很高, 达到 $314.97 \mathrm{mg} / \mathrm{kg}$. 而在运动营 养学中提到, 非运动员每日对 $\mathrm{VC}$ 需要量是 $75 \mathrm{mg} / \mathrm{kg}$, 速度 运动员和力量运动员每日对 $\mathrm{VC}$ 需要则是 $100-200 \mathrm{mg} / \mathrm{kg}$. 耐 力运动员每日对 $\mathrm{VC}$ 的需要则是 $100-300 \mathrm{mg} / \mathrm{kg}$, 可见从运动 营养学角度看, 玛卡对于运动员补充 $\mathrm{VC}$ 是很有帮助。

\subsection{1 玛卡氨基酸的运动营养学分析:}

表 2 云南玛卡中氨基酸分析结果

\begin{tabular}{|c|c|}
\hline 氨基酸 & 含量 $/ \%$ \\
\hline 天门冬氨酸 & 1.00 \\
\hline 苏氨酸 & 0.48 \\
\hline 丝氨酸 & 0.46 \\
\hline 谷氨酸 & 2.00 \\
\hline 甘氨酸 & 0.46 \\
\hline 丙氨酸 & 0.47 \\
\hline 异亮氨酸 & 0.44 \\
\hline 胱氨酸 & 0.09 \\
\hline 倾氨酸 & 0.66 \\
\hline 蛋氨酸 & 0.05 \\
\hline 组氨酸 & 0.35 \\
\hline 精氨酸 & 3.48 \\
\hline 苯丙氨酸 & 0.40 \\
\hline 亮氨酸 & 0.54 \\
\hline 酪氨酸 & 0.29 \\
\hline 赖氨酸 & 0.82 \\
\hline 脯氨酸 & 2.05 \\
\hline 氨基酸总量 & 14.04 \\
\hline
\end{tabular}

营养学提出“必需氨基酸指的是人体自身不能合成或 合成速度不能满足人体需要, 必须从食物中摄取的氨基酸。 它是人体 (或其它脊椎动物) 必不可少, 而机体内又不有 合成的, 必须从食物中补充的氨基酸。而营养学界公认人 体必需氨基酸共有八种: 赖氨酸、色氨酸、苯丙氨酸、甲 硫氨酸 (蛋氨酸) 、苏氨酸、异亮氨酸、亮氨酸、坃氨酸, 而在对玛卡的氨基酸分析中我们可以发现, 玛卡含有 17 种 氨基酸其中必需氨基酸 7 种, 氨基酸总量达到 $14.04 \%$ 。大 家知道运动生理学提出人体运动时骨骼肌内三个供能系统 中, 磷酸原供能供能系统中磷酸肌酸 ( $\mathrm{cp}$ ) 是肌酸 (C) 的 磷酸化形式。肌酸由精氨酸、甘氨酸, 甲硫氨酸 (蛋氨酸) 合成。而在玛卡的氨基酸营养成分中, 精氨酸占 (3.48\%) 甘氨酸 $(0.46 \%)$ 甲硫氨酸 (蛋氨酸) 占 $(0.05 \%)$ 。这三种 氨基酸占玛卡氨基酸总量的 $28.41 \%$, 其运动营养学价值很
高。在运动饮食中适量饮用和食用玛卡, 可以为运动员提 供充足的蛋白质。

2.3 云南玛卡中矿物质的运动营养学分析

2.3.1 云南玛卡矿物质分析结果

表 3 云南玛卡中矿物元素分析结果

\begin{tabular}{|c|c|c|c|c|c|}
\hline 微量元素 & $\mathrm{Fe}$ & $\mathrm{Ca}$ & $\mathrm{Zn}$ & $\mathrm{Na}$ & $\mathrm{Cu}$ \\
\hline 含量 $/(\mathrm{mg} / \mathrm{kg})$ & 81.2 & 1818 & 23.8 & 182 & 4.25 \\
\hline 微量元素 & $\mathrm{Cd}$ & $\mathrm{Mg}$ & $\mathrm{K}$ & $\mathrm{P}$ & $\mathrm{Mn}$ \\
\hline 含量 $/(\mathrm{mg} / \mathrm{kg})$ & 0.41 & 798 & 18.7 & 1895 & 13.2 \\
\hline
\end{tabular}

表 4 运动员的矿物质需要量

\begin{tabular}{|c|c|c|c|c|c|}
\hline & 钾 $/ \mathrm{g}$ & 氯化钠 $/ \mathrm{g}$ & 钻 $/ \mathrm{g}$ & 镁 $/ \mathrm{mg}$ & 铁 \\
\hline 训练期 & 3 & 15 & 0.8 & $300-500$ & 15 \\
\hline 比赛期 & $4-6$ & $20-25$ & $1-1.5$ & $500-800$ & $20-25$ \\
\hline
\end{tabular}

2.3.2 玛卡矿物质成分在运动营养中分析

2.3.3 通过表 3 分析, 云南玛卡检测出 11 种矿物质微量元 素。其中 $\mathrm{P}$ 的含量最高达到 $1895 \mathrm{mg} / \mathrm{kg}$, 其次是 $\mathrm{Ca}$ 为 $1818 \mathrm{mg} / \mathrm{kg}$ 和 $\mathrm{Mg}$ 为 $798 \mathrm{mg} / \mathrm{kg}$.

2.3.4 P 是构成骨与牙齿的主要成分, 磷是在物质代谢中有 着重要作用。

1. P 参与物质能量代谢

2. $\mathrm{P}$ 与脂肪合成磷脂 (是构成神经组织重要物质)

3. 维持血液的酸碱平衡

4. 磷酸盐的补充加强体内磷酸化过程, 可以改善运动 能力。

$\mathrm{P}$ 是运动员膳食中重要的营养素之一。运动营养学认 为成人每天所需 $\mathrm{P}$ 为 $1.5 \mathrm{~g}$, 运动员每日所需为 $2.0-2.5 \mathrm{~g}$, 在 能量消耗大或者神经紧张情况下, 每日所需 $\mathrm{P}$ 为 3.0-4.5g, 而在玛卡中拍 $\mathrm{P}$ 的含量为 $1.895 \mathrm{~g}$

2.3.5 Ca 构成骨骼和牙齿, 缺钻时容易神经肌肉的应激性增 高, 肌肉容易发生痉挛。目前我国成人一般训练为 $0.8 \mathrm{~g} / \mathrm{d}$, 比赛其为 $1-1.5 \mathrm{~g} / \mathrm{d}$. 而玛卡的 $\mathrm{Ca}$ 为 $1.818 \mathrm{~g} / \mathrm{d}$, 如果在运动员平 时的营养膳食中添加玛卡, 可以补充训练所需的 $\mathrm{Ca}$.

2.3.6 运动生理学认为运动员对微量元素和矿物质需求较 多, 一方面在运动时体内代谢加强, 激素分泌与酶的活动 增强, 加上大量出汗, 物质流失很多。另一方面, 体内充 足矿物质和微量元素储备, 可以改善运动员运动机体的能 力, 并提高运动员运动成绩。 
表 5 云南玛卡脂肪酸的化学成分

\begin{tabular}{|c|c|c|c|c|c|c|c|c|c|c|}
\hline 保留时间 $/ \mathrm{min}$ & 2.472 & 2.963 & 3.295 & 5.287 & 6.914 & 7.173 & 7.244 & 7.715 & 8.533 & 9.302 \\
\hline 相对含量 $/ \%$ & 0.95 & 1.88 & 0.79 & 17.72 & 1.67 & 3.04 & 7.58 & 38.64 & 26.46 & 1.26 \\
\hline 脂肪酸名称 & 辛酸 & 未知 & 月桂酸 & 棕㭣酸 & 硬脂酸 & 未知 & 油酸 & 亚油酸 & 亚麻酸 & 花生四烯酸 \\
\hline
\end{tabular}

\subsection{2 云南玛卡中脂肪酸在运动营养中分析}

\subsection{3 脂肪酸对人体重要作用:}

必需脂肪酸 (essential fatty acids, EFA) 是指人体维持 机体正常代谢不可缺少而自身又不能合成、或合成速度慢 无法满足机体需要, 必须通过食物供给的脂肪酸。必需脂 肪酸不仅能够吸引水分滋润皮肤细胞, 还能防止水分流失。 它是机体润滑油, 但人体自身不能合成, 必须从食物中摄 取. n-6 系列的亚油酸和 n-3 系列的亚麻酸是人体必需的两 种脂肪酸。它们都是多不饱和脂肪酸, 其中以亚油酸最为 重要, 它在一定程度上可以替代和节约亚麻酸。事实上, $n-6 n-3$ 系列中许多脂肪酸如花生四烯酸 (AA)、二十碳五 烯酸 (EPA)、二十二碳六烯酸 (DHA) 等都是人体不可缺 少的, 但人体可利用亚油酸和 $\alpha$-亚麻酸合成这些脂肪酸。

2.4.4 n-3 在运动训练中的作用: 1. 促进氧的运输, 对有氧运 动能力提高有好处。2. 增强心肌收缩能力 3 . 增强运动员反 应能力 4.DHA 对记忆功能很重要。而在玛卡的脂肪酸化学 反应数据中可以看到, $\mathrm{n}-3$ 系列的亚麻酸占到 $38.64 \%$ 说明 云南玛卡中亚油酸的含量是很高的, 在运动营养中的应用 价值很强。在运动员饮食中适量食用玛卡, 可以很好的补 充其身体对亚油酸的需要。

\section{3. 玛卡的食用特性在运动营养学中的作用}

由于玛卡中含有大量的钠钾钙镁等金属, 所以玛卡 是碱性食物。运动生理学认为: 由于剧烈的运动可造成机 体内酸性代谢产物的堆积, 使运动员体内酸性偏高, 因此 在运动员营养补充过程中多摄入碱性食物, 以利体内酸碱 储备, 对运动员能力提高有良好作用。所以在运动膳食营 养中食用玛卡对运动能力提高是有益的。

\section{4. 结论与分析}

4.1 通过对云南玛卡的主要营养成分的分析, 表明了玛卡具 有很强的营养学价值。其富含的蛋白质、碳水化合物、热 量、 $\mathrm{VC}$ 、粗纤维、多种人体所需氨基酸、矿物质、脂肪酸 等物质, 是运动员在平时训练和比赛过程中身体机能所必 需要的基础物质能量补充。

4.2 玛卡不仅有很强的营养价值, 还含有很多活性酶。例如: 生物碱, 玛卡烯等活性酶, 可以很好的提高运动员的免疫 力和快速恢复身机能能力, 并且还能有效缓解运动员训练 和比赛中运动性疲劳问题。

\section{参考文献(References)}

[1] 1 Yu Longjiang, Jin Wen. Study and development, botany and pharmacological studies of. natural product Maca 2002, 14 (5): 24-27

[2] Analysis of nutritional components of 2 Yunnan Maca Du Ping, a single cloud sun Hui, Sun Xiaodong, Qu Xu, Zhang Xianjun, Yao Min. 1. Kunming University of Science and Technology center of analysis and test research, analysis and test center in Yunnan Province, Yunnan Kunming 650093; 2. Huize County Hongren Trade Industry Co. Ltd, Yunnan Huize 654200.

[3] Li Zhixiang Shen Cuiping polyunsaturated fatty acid on human J biology bulletin 1988, 33 (1), 9-103

[4] Xiong Zhengying Zhang Chongxu Zheng Yi polyunsaturated fatty acids and Shaanxi Normal University Sports Institute of Biology 